\title{
Calculation of the Absorption Cross Sections of Some Molecules from GEISA Database at the Wavelengths of Isotopically Different $\mathrm{CO}_{2}$ Lasers
}

\author{
Asylkhan Rakhymzhan ${ }^{1}$ and Alexey Chichinin ${ }^{1,2}$ \\ ${ }^{1}$ Institute of Chemical Kinetics and Combustion, Novosibirsk 630090, Russia \\ ${ }^{2}$ Novosibirsk State University, Novosibirsk 630090, Russia \\ Correspondence should be addressed to Alexey Chichinin; chichinin@kinetics.nsc.ru
}

Received 8 July 2013; Accepted 19 September 2013

Academic Editors: J. A. Lopes and Y. van der Burgt

Copyright ( 2013 A. Rakhymzhan and A. Chichinin. This is an open access article distributed under the Creative Commons Attribution License, which permits unrestricted use, distribution, and reproduction in any medium, provided the original work is properly cited.

A calculation of the absorption cross section of some molecules $\left(\mathrm{NH}_{3}, \mathrm{C}_{2} \mathrm{H}_{4}, \mathrm{CO}_{2}, \mathrm{O}_{3}, \mathrm{NO}_{2}, \mathrm{PH}_{3}, \mathrm{HNO}_{3}, \mathrm{SF}_{6}, \mathrm{CH} \mathrm{OH}_{3} \mathrm{HCOOH}\right.$, OCS, $\mathrm{CH}_{3} \mathrm{CN}, \mathrm{C}_{2} \mathrm{H}_{6}, \mathrm{SO}_{2}$, and $\left.\mathrm{H}_{2} \mathrm{O}\right)$ at the wavelengths transmitted by a $\mathrm{CO}_{2}$ laser filled with different isotopes $\left({ }^{12} \mathrm{C}^{16} \mathrm{O}_{2},{ }^{13} \mathrm{C}^{16} \mathrm{O}_{2}\right.$, ${ }^{12} \mathrm{C}^{18} \mathrm{O}_{2},{ }^{14} \mathrm{C}^{16} \mathrm{O}_{2},{ }^{14} \mathrm{C}^{18} \mathrm{O}_{2},{ }^{13} \mathrm{C}^{18} \mathrm{O}_{2}$, and ${ }^{12} \mathrm{C}^{16} \mathrm{O}^{18} \mathrm{O}$ ) is presented. The spectroscopical parameters for the molecules from GEISA database have been used. Hence the selection of the molecules was substantially based on the availability of the parameters in the database. The results of the calculations may be used in designing the differential absorption technique for remote monitoring of these molecules. The pressure and temperature dependence of the cross sections are described by $K_{T}$ and $K_{P}$ coefficients; these coefficients were calculated for the largest absorption cross sections for each molecule. The absorption cross sections of $\mathrm{CH}_{3} \mathrm{OH}$ and $\mathrm{HCOOH}$ at low pressures for all these $\mathrm{CO}_{2}$ lasers are also presented. These calculations are provided for design of new $\mathrm{CO}_{2}-$ laser-pumped far-infrared lasers.

\section{Introduction}

In this paper we report molecular absorption cross sections $\sigma(\mathrm{M})$ at $\mathrm{CO}_{2}$-laser emission frequencies for several selected gases of atmospheric relevance $\left(\mathrm{M}=\mathrm{NH}_{3}, \mathrm{C}_{2} \mathrm{H}_{4}, \mathrm{CO}_{2}, \mathrm{O}_{3}\right.$, $\mathrm{NO}_{2}, \mathrm{PH}_{3}, \mathrm{HNO}_{3}, \mathrm{SF}_{6}, \mathrm{CH}_{3} \mathrm{OH}, \mathrm{HCOOH}, \mathrm{OCS}, \mathrm{CH}_{3} \mathrm{CN}$, $\mathrm{C}_{2} \mathrm{H}_{6}, \mathrm{SO}_{2}$, and $\mathrm{H}_{2} \mathrm{O}$ ). This information may be useful mainly in the differential absorption (Light Detection and Ranging) LIDAR technique for remote measurement of the gas species [1-8] and also may be used to monitor the $\mathrm{CO}_{2}$ content in fuel combustion products [9], remote sensing of gases in human breath [10], or multiphoton dissociation processes or to measure water vapor concentration and wind speed vector in the plume of volcano $[11,12]$. Note that the LIDAR technique sometimes is used for remote sensing of some exotic gases, like, for example, chemical warfare [13].

In some cases $\left(\mathrm{CH}_{3} \mathrm{OH}\right.$ and $\left.\mathrm{HCOOH}\right)$ it also may be used in designing optically pumped FIR (far infrared $=\mathrm{THz}$ ) lasers where $\mathrm{CO}_{2}$ laser is used as a source of a pump radiation [14].
Also, the absorption of $\mathrm{CO}_{2}$-laser radiation by a cell with a mixture of some of these gases is used in our lab for quick check and assignment of the $\mathrm{CO}_{2}$-laser lines.

The focus of the present study is to predict absorption cross section in pure air at wavelengths of seven isotopic $\mathrm{CO}_{2}$ lasers: ${ }^{12} \mathrm{C}^{16} \mathrm{O}_{2}$ (normal), ${ }^{13} \mathrm{C}^{16} \mathrm{O}_{2},{ }^{12} \mathrm{C}^{18} \mathrm{O}_{2},{ }^{14} \mathrm{C}^{16} \mathrm{O}_{2}$, ${ }^{14} \mathrm{C}^{18} \mathrm{O}_{2},{ }^{13} \mathrm{C}^{18} \mathrm{O}_{2}$, and ${ }^{12} \mathrm{C}^{16} \mathrm{O}^{18} \mathrm{O}$, which we hereafter denote as 26-, 36-, 28-, 46-, 48-, 38-, and 268-lasers.

In the clear atmosphere, absorption at $9-11 \mu \mathrm{m}$ is due primarily to water vapor and carbon dioxide. Since the fraction of $\mathrm{CO}_{2}$ in the atmosphere is about $3.8 \times 10^{-4}$ and $\sigma\left(\mathrm{CO}_{2}\right) \sim$ $10^{-22} \mathrm{~cm}^{2}$, the resonant absorption of $50 \%$ of the 26-laser radiation by atmospheric molecules occurs at the distance about $7 \mathrm{~km}$. This distance may be not large enough for typical LIDAR applications, like monitoring of air pollution over the large town or early detection of small forest fires [15, 16]. Also, the fluctuations of the $\mathrm{CO}_{2}$ concentration in the atmosphere decrease strongly the accuracy of the LIDAR based on the 26-laser. Hence the first advantage of latter six 
$\mathrm{CO}_{2}$ lasers over conventional 26-laser is low attenuation from atmospheric $\mathrm{CO}_{2}$, which may extend strongly the detection distance and/or the accuracy of the LIDAR. Moreover, these isotopically substituted $\mathrm{CO}_{2}$ lasers may be used to detect the concentration of $\mathrm{CO}_{2}$ in the atmosphere or, more simply, to monitor the $\mathrm{CO}_{2}$ content in fuel combustion products [9].

Another advantage is another set of wavelengths; sometimes it makes it possible to detect molecules, unavailable for conventional 26-laser LIDAR.

It is important to note that the use of $\mathrm{CO}_{2}$ lasers for LIDAR remote sensing offers some advantages over that of the more current LIDAR experiments being conducted using the fundamental absorption transitions of hydrocarbons near 3 to $4 \mu \mathrm{m}$ and CW Quantum Cascade Lasers, in that the absorption values near $10 \mu \mathrm{m}$ are not too high and not too low for remote sensing at ranges of a few hundred meters, and the output power of the $\mathrm{CO}_{2}$ laser is high offering good detection ranges.

Note that commercially available $\mathrm{CO}_{2}$ lasers often may be filled with different isotopic gases (e.g., PL3 series from Edinburgh Photonics). Also, $\mathrm{CO}_{2}$ lasers make up to $10^{8}$ shots without changing of the gas mixture (e.g., InfraLight series of $\mathrm{CO}_{2}$ lasers); hence there is no large difference, which isotopic modification of $\mathrm{CO}_{2}$ gas to use.

This work was greatly facilitated by usage of GEISA spectroscopical database [17], and only the molecules from the database were involved in calculations. We have not included several gases $\left(\mathrm{N}_{2} \mathrm{H}_{4}, \mathrm{C}_{6} \mathrm{H}_{6}, \mathrm{C}_{2} \mathrm{Cl}_{4}, \mathrm{C}_{2} \mathrm{HCl}_{3}, \mathrm{C}_{2} \mathrm{H}_{3} \mathrm{Cl}\right.$, $\mathrm{C}_{2} \mathrm{H}_{5} \mathrm{SH}, \mathrm{C}_{2} \mathrm{H}_{4} \mathrm{Cl}_{2}, \mathrm{CF}_{2} \mathrm{Cl}_{2}$, and $\mathrm{CFCl}_{3}$ ) which may be detected by $\mathrm{CO}_{2}$ lasers [18], since no information is available on their absorption cross sections in the GEISA and HITRAN databases.

There were a lot of experimental measurements of the absorption coefficients at 26-laser frequencies. The most popular molecules are $\mathrm{SF}_{6}[19-21], \mathrm{C}_{2} \mathrm{H}_{4}$ [22-24], and $\mathrm{NH}_{3}$ [22-26], and the list of references here is very large. A lot of $\sigma(\mathrm{M})$ for more complicated molecules are also reported in the literature, for example, for acetonitrile, benzene, cyclohexane, 1,2-dichloroethane, ethyl acetate, freon-12, freon113, furan, isopropanol, methyl chloroform, methyl ethyl ketone, $t$-butanol, vinyl chloride, and iodopropane [27]; seven hydrazine fuel gases [28]; hydrazine, unsymmetrical dimethylhydrazine, and monomethylhydrazine [29]; $\mathrm{C}_{2} \mathrm{H}_{4}$, $\mathrm{C}_{2} \mathrm{H}_{3} \mathrm{Cl}_{3}, \mathrm{C}_{2} \mathrm{HCl}_{3}$, and Freon-113 [3]; and triacetone triperoxide [8].

However, much less information is available regarding $\sigma(\mathrm{M})$ of $\mathrm{CO}_{2}$ lasers other than 26-laser. For example, we know the measurements of $\sigma\left(\mathrm{H}_{2} \mathrm{O}\right)$ at 26-, 36-, and 46-laser wavelengths [30], $\sigma\left(\mathrm{ClO}_{2}\right)$ at 28-laser wavelengths [31], $\sigma\left(\mathrm{NH}_{3}\right)$ at a 36-laser [32]. Also, the photoacoustic spectroscopy has been used to determine $\sigma(\mathrm{M})$ for $\mathrm{M}=\mathrm{NH}_{3}, \mathrm{CCl}_{2} \mathrm{~F}_{2}, \mathrm{CHClF}_{2}$, $\mathrm{CFCl}_{3}$, and $\mathrm{CClF}_{3}$ at 36-laser wavelengths [33].

To the best of our knowledge, the FIR lasers normally are pumped by 26-lasers or, much rarely, by 36-laser [3437]; the other $\mathrm{CO}_{2}$ lasers are used very rarely [38, 39]. Note that while 26-laser has about 100 laser lines, using different isotopic $\mathrm{CO}_{2}$ lasers gives up to 1000 lines; hence the amount of different FIR-lasers pumped by $\mathrm{CO}_{2}$ lasers should increase accordingly.
With this in mind, we performed the calculation which hopefully provides the information of the quality comparable with that of the experimental studies. We hope that it will stimulate using isotopically different $\mathrm{CO}_{2}$ lasers for different applications.

\section{Results}

Assuming Lorentzian line shapes, we calculated the absorption cross sections $\sigma(\mathrm{M})$ at all possible $\mathrm{CO}_{2}$-laser frequencies. Tables 1, 2, and 3 show $\sigma(\mathrm{M})$ for 26-, 36-, and 28-lasers, respectively, for $\mathrm{CO}_{2}$-laser lines between $\mathrm{P}(40)$ and $\mathrm{R}(40)$, excluding the range $\mathrm{P}(6)-\mathrm{R}(6)$. Atmospheric pressure $P_{0}=1$ bar and temperature $T_{0}=296 \mathrm{~K}$ are assumed everywhere, and the self-broadening is neglected (i.e., $[\mathrm{M}] \ll[$ air $]$ ). The following expressions were used:

$$
\begin{gathered}
\sigma(\mathrm{M})=\sum_{i} \frac{S_{i}(T)}{\pi} \frac{\Delta v_{i}(P, T)}{\Delta v_{i}^{2}(P, T)+\left(\nu-v_{i}-\delta_{i} P\right)^{2}}, \\
S_{i}(T)=S_{i 0}\left(\frac{T_{0}}{T}\right)^{L} \exp \left(-\frac{E_{i}}{k T}+\frac{E_{i}}{k T_{0}}\right), \\
\Delta v_{i}(P, T)=\Delta v_{i 0}\left(\frac{P}{P_{0}}\right)\left(\frac{T_{0}}{T}\right)^{m_{i}},
\end{gathered}
$$

where the index $i$ labels all transitions in molecule $\mathrm{M}, S_{i}(T)$ is intensity of the $i$ th spectral line, $S_{i 0}=S_{i}\left(T_{0}\right), \Delta v_{i}(P, T)$ is Lorentzian width, $\Delta v_{i 0}=\Delta v_{i}\left(P_{0}, T_{0}\right), v_{i}$ is the absorption maximum frequency of the $i$ th spectral line, $\delta_{i}$ is the pressure shift of the line transition, $E_{i}$ is the energy of the lower state for $i$ th transition, $L=1$ for linear molecules like $\mathrm{CO}_{2}$ and $L=3 / 2$ for nonlinear molecules, $P$ and $T$ are air pressure and temperature, respectively, and $k$ is Boltzmann constant.

The parameters $v_{i}, S_{i 0}, \Delta v_{i 0}, m_{i}, \delta_{i}$, and $E_{i}$ are taken from GEISA database for each transition of each $\mathrm{M}$ molecule. Note that the pressure shifts $\delta_{i}$ are given in the database only for $\mathrm{CH}_{3} \mathrm{CN}$ and $\mathrm{NO}_{2}$ molecules. All of them are rather small $\left(\approx 10^{-3} \mathrm{~cm}^{-1} / \mathrm{atm}\right)$, and they change only the third digit in calculated absorption cross sections. We hope that there is the same situation with all other molecules; hence we present the cross sections with three-digit accuracy; the last digit may be wrong due to the pressure shifts. All $\mathrm{CO}_{2}$-laser frequencies were taken from Freed et al. [40].

In Table 4 we present the "best" laser transitions for each isotopic variation of $\mathrm{CO}_{2}$ laser and for each molecule $\mathrm{M}$. The pressure and temperature dependence of the cross sections are described by $K_{T}$ and $K_{P}$ coefficients as $K_{T}=d \ln \sigma / d \ln T$, $K_{P}=d \ln \sigma / d \ln T$, and the coefficients have been calculated from (1) and presented in Table 4 also.

It is not easy task to point out the "best" $\mathrm{CO}_{2}$-laser line for detection of molecule $\mathrm{M}$. Normally the "best" $\mathrm{CO}_{2}$-laser line should lie in the ranges $\mathrm{R}(10)-\mathrm{R}(40), \mathrm{P}(10)-\mathrm{P}(40)$ and has the largest absorption by $\mathrm{M}$ molecules; if the largest $\sigma(\mathrm{M})$ values occur outside these ranges, we mark it by asterisk shown in the table also. However, if the largest $\sigma(\mathrm{M})$ values occur at marginal lines of $\mathrm{CO}_{2}$ laser and are much larger than all other cross sections, we present this marginal line only. 


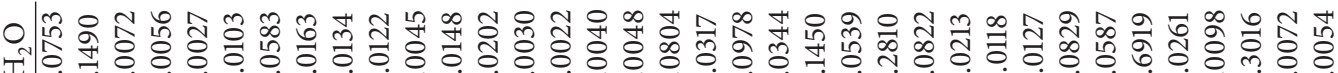
$x^{2}$

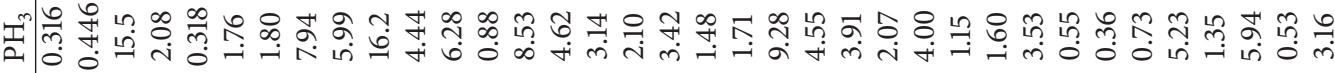

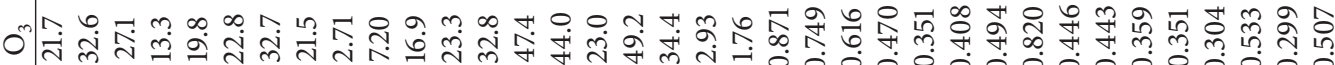
i⿱宀

으

ซั

恄

$\stackrel{0}{4}$

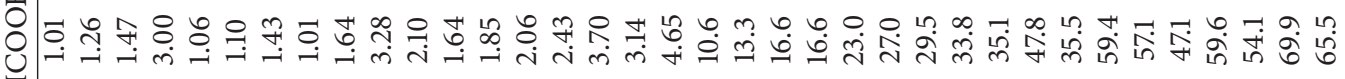
코

مَ

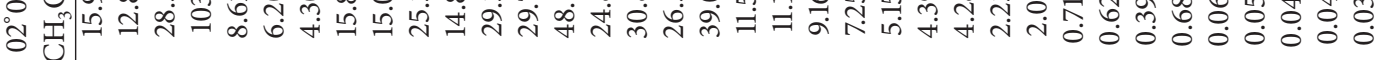
$\uparrow$

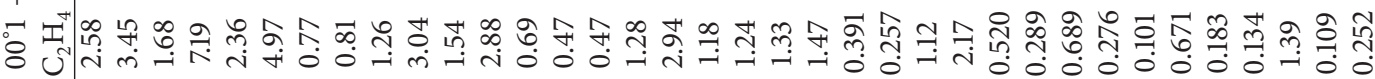

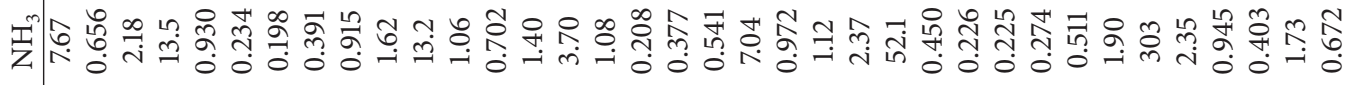

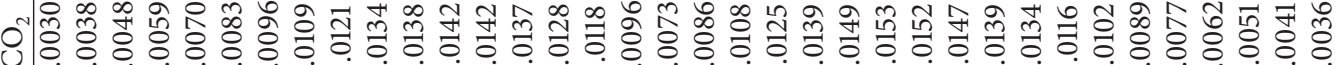

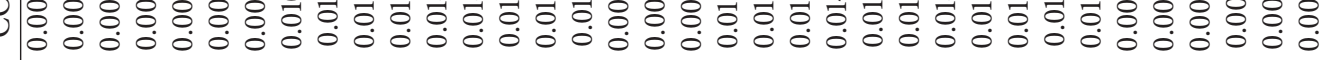

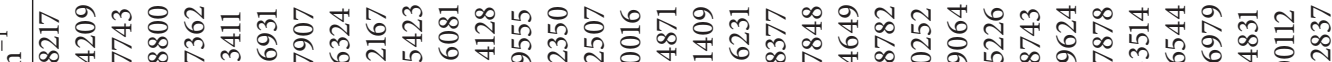

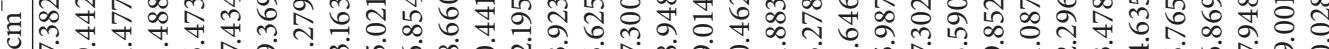

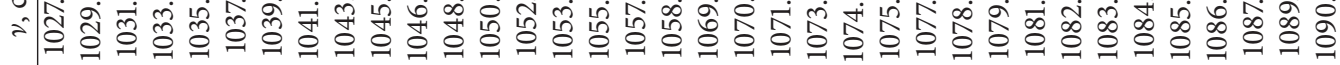

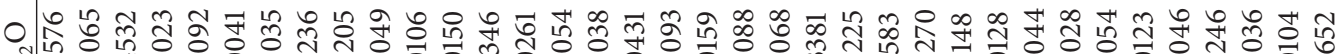

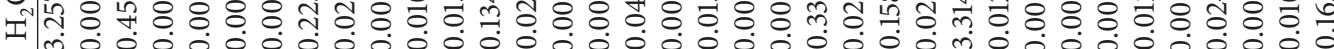

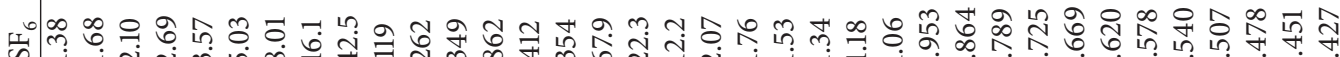
(1) 方旗

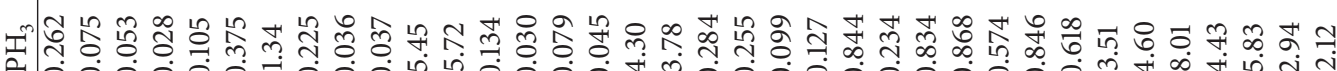

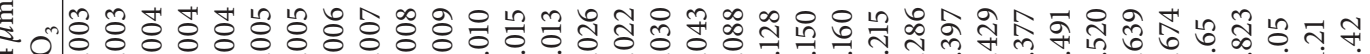
$\cong$

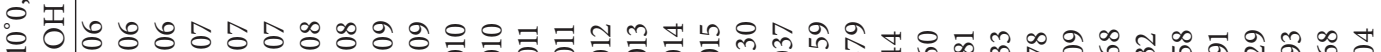

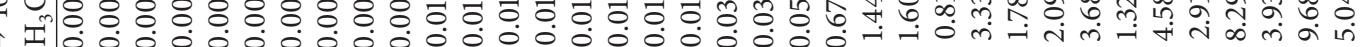
8 Ұ जी



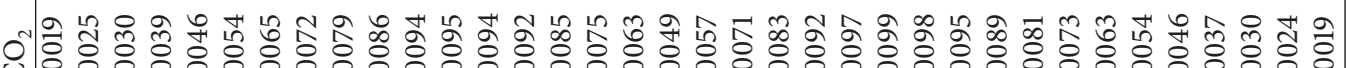
u Т

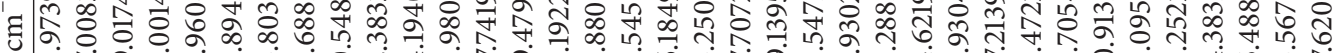

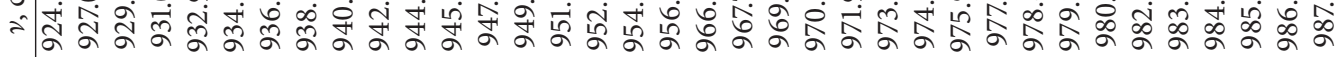

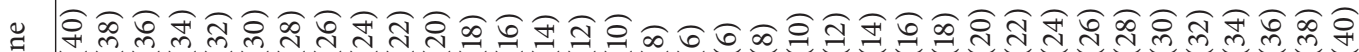

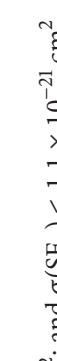




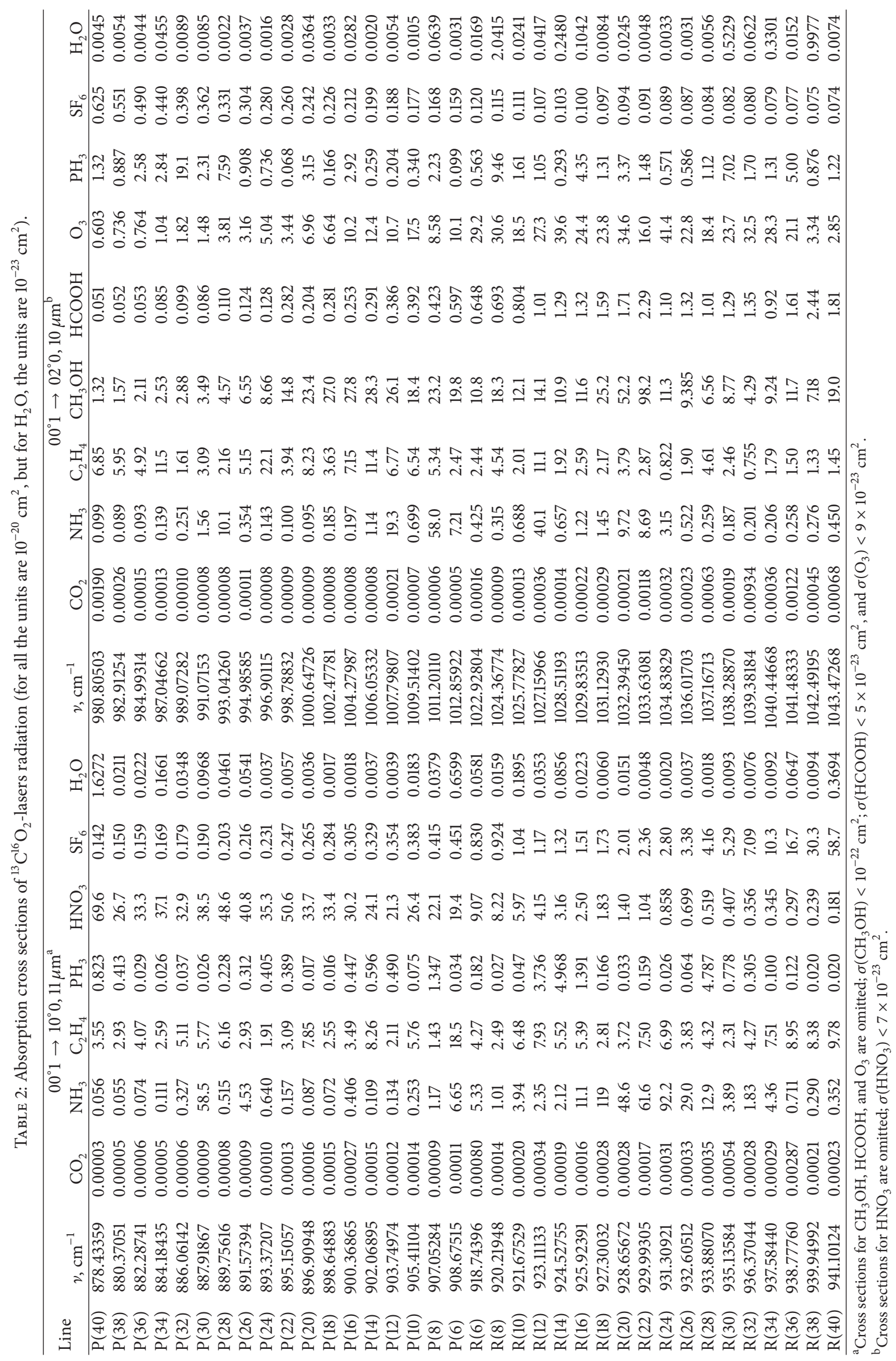




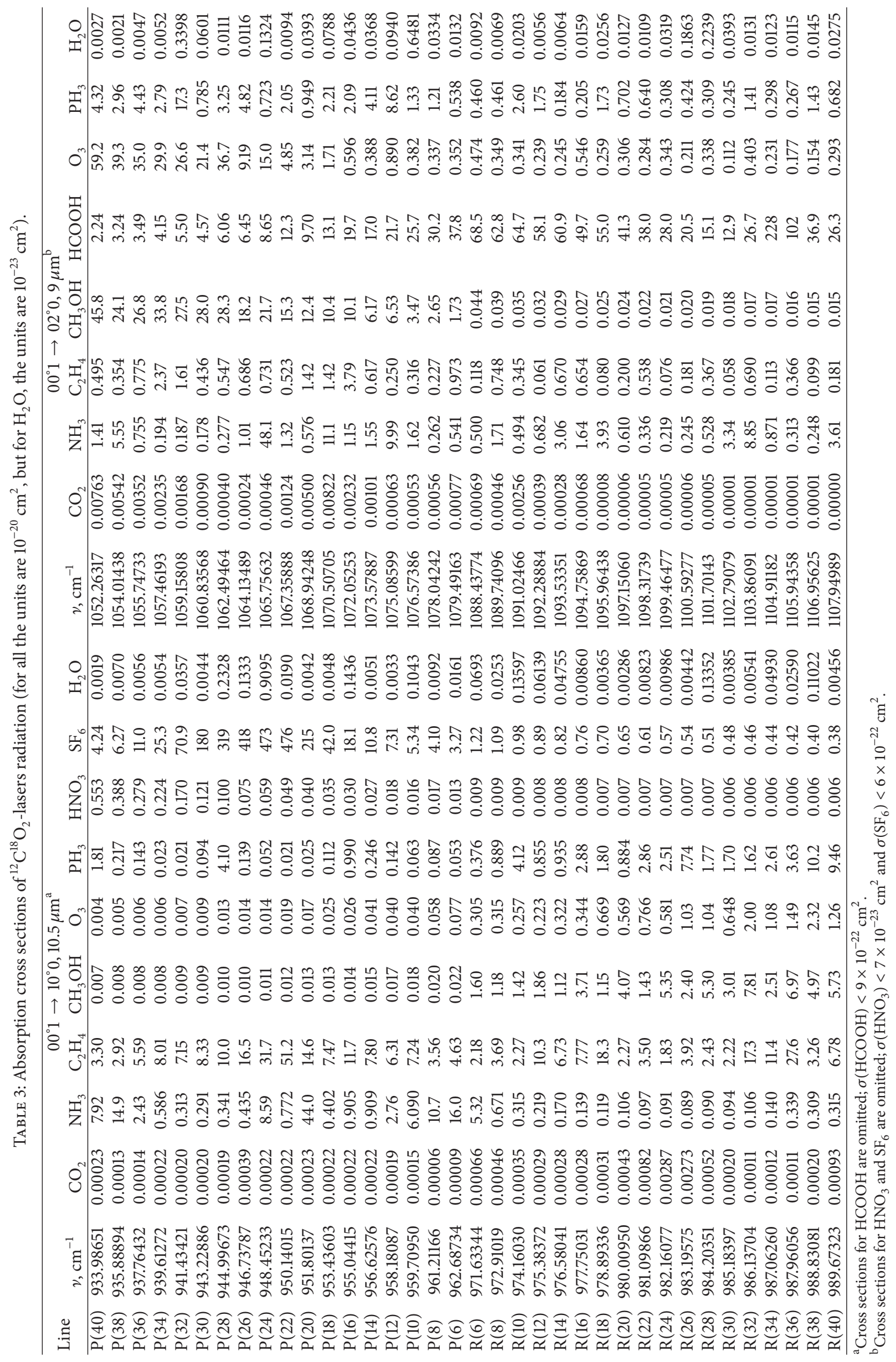


TABLE 4: For each $\mathrm{CO}_{2}$ laser and for each molecule $\mathrm{M}$, the laser transitions with the largest absorption by selected molecules are presented. The cross sections $\sigma(\mathrm{M})$ (in $10^{-20} \mathrm{~cm}^{2}$ units) and $K_{T}$ and $K_{p}$ coefficients are shown.

\begin{tabular}{|c|c|c|c|c|c|c|}
\hline Laser & M & Transition & $v, \mathrm{~cm}^{-1}$ & $\sigma(\mathrm{M})^{\mathrm{a}}$ & $K_{T}$ & $K_{P}$ \\
\hline \multirow{15}{*}{26} & $\mathrm{NH}_{3}$ & $10 \mathrm{R}(30)$ & 1084.63514 & 303 & 0.38 & -0.83 \\
\hline & $\mathrm{C}_{2} \mathrm{H}_{4}$ & $11 \mathrm{P}(14)$ & 949.47931 & 133 & 0.16 & -0.13 \\
\hline & $\mathrm{O}_{3}$ & $10 \mathrm{P}(14)$ & 1052.19555 & 47.4 & -0.62 & 0.08 \\
\hline & & $10 \mathrm{P}(8)$ & 1057.30016 & $49.2^{*}$ & -0.02 & -0.12 \\
\hline & $\mathrm{NO}_{2}$ & $11 \mathrm{P}(48)$ & 916.58177 & 0.143 & 3.11 & -0.36 \\
\hline & $\mathrm{PH}_{3}$ & $10 \mathrm{P}(22)$ & 1045.02167 & 16.2 & -0.19 & -0.46 \\
\hline & $\mathrm{HNO}_{3}$ & $11 \mathrm{P}(44)$ & 920.82912 & 6.89 & 1.90 & -0.02 \\
\hline & $\mathrm{SF}_{6}$ & $11 \mathrm{P}(16)$ & 947.74198 & 862 & -0.02 & -0.58 \\
\hline & $\mathrm{CH}_{3} \mathrm{OH}$ & $10 \mathrm{P}(34)$ & 1033.48800 & 103 & 0.44 & -0.05 \\
\hline & $\mathrm{HCOOH}$ & $10 \mathrm{R}(28)$ & 1083.47878 & 59.4 & 0.37 & -0.22 \\
\hline & OCS & $10 \mathrm{P}(32)$ & 1035.47362 & 1.68 & -0.13 & -0.14 \\
\hline & $\mathrm{CH}_{3} \mathrm{CN}$ & $10 \mathrm{R}(16)$ & 1075.98782 & 1.82 & 1.38 & -0.30 \\
\hline & $\mathrm{SO}_{2}$ & $10 \mathrm{R}(26)$ & 1082.29624 & 0.430 & 2.26 & -0.31 \\
\hline & $\mathrm{SO}_{2}$ & $10 \mathrm{R}(40)$ & 1090.02837 & $0.595^{*}$ & 1.54 & 0.15 \\
\hline & $\mathrm{H}_{2} \mathrm{O}$ & $11 \mathrm{R}(20)$ & 975.93044 & 0.00331 & 6.47 & -0.85 \\
\hline \multirow{16}{*}{36} & $\mathrm{NH}_{3}$ & 11R(18) & 927.30032 & 119 & 0.57 & -0.87 \\
\hline & $\mathrm{C}_{2} \mathrm{H}_{4}$ & $10 \mathrm{P}(24)$ & 996.90115 & 22.1 & -0.14 & -0.44 \\
\hline & $\mathrm{CO}_{2}$ & $10 \mathrm{R}(32)$ & 1039.38184 & 0.009 & 7.45 & -0.93 \\
\hline & $\mathrm{O}_{3}$ & $10 \mathrm{R}(24)$ & 1034.83829 & 41.4 & -0.02 & -0.42 \\
\hline & $\mathrm{NO}_{2}$ & $11 \mathrm{P}(38)$ & 880.37051 & 0.461 & 1.43 & -0.25 \\
\hline & $\mathrm{PH}_{3}$ & $10 \mathrm{P}(32)$ & 989.07282 & 19.1 & 0.92 & -0.21 \\
\hline & $\mathrm{HNO}_{3}$ & $11 \mathrm{P}(22)$ & 895.15057 & 50.6 & 0.36 & -0.01 \\
\hline & & $11 \mathrm{P}(40)$ & 878.43359 & $69.6^{*}$ & 0.26 & 0.05 \\
\hline & $\mathrm{SF}_{6}$ & $11 \mathrm{R}(42)$ & 942.23141 & 110 & 0.87 & 0.14 \\
\hline & & $11 \mathrm{R}(44)$ & 943.34030 & $189^{*}$ & 0.38 & 0.02 \\
\hline & $\mathrm{CH}_{3} \mathrm{OH}$ & $10 \mathrm{R}(22)$ & 1033.63081 & 98.2 & 0.45 & -0.07 \\
\hline & $\mathrm{HCOOH}$ & $10 \mathrm{R}(38)$ & 1042.49195 & 2.44 & 1.22 & -0.23 \\
\hline & OCS & $10 \mathrm{R}(36)$ & 1041.48333 & 2.08 & -0.14 & -0.50 \\
\hline & $\mathrm{CH}_{3} \mathrm{CN}$ & $10 \mathrm{R}(10)$ & 1025.77827 & 2.86 & 0.23 & 0.01 \\
\hline & $\mathrm{C}_{2} \mathrm{H}_{6}$ & $11 \mathrm{P}(48)$ & 870.48389 & 0.493 & 0.85 & 0.06 \\
\hline & $\mathrm{H}_{2} \mathrm{O}$ & $9 \mathrm{R}(8)$ & 1024.36774 & 0.00204 & 6.41 & 0.65 \\
\hline \multirow{19}{*}{28} & $\mathrm{NH}_{3}$ & $11 \mathrm{P}(20)$ & 951.80137 & 44.0 & -0.89 & -0.88 \\
\hline & & $11 \mathrm{P}(42)$ & 932.05695 & $67.9^{*}$ & -0.37 & -0.27 \\
\hline & $\mathrm{C}_{2} \mathrm{H}_{4}$ & $11 \mathrm{P}(22)$ & 950.14015 & 51.2 & 0.49 & 0.04 \\
\hline & $\mathrm{CO}_{2}$ & $10 \mathrm{P}(18)$ & 1070.50705 & 0.008 & 5.22 & -0.50 \\
\hline & & $10 \mathrm{P}(44)$ & 1048.70641 & $0.010^{*}$ & 6.19 & -0.42 \\
\hline & $\mathrm{O}_{3}$ & $10 \mathrm{P}(40)$ & 1052.26317 & $59.2^{*}$ & -0.38 & -0.38 \\
\hline & & $10 \mathrm{P}(36)$ & 1055.74733 & 35.0 & -0.10 & 0.04 \\
\hline & $\mathrm{NO}_{2}$ & $11 \mathrm{P}(42)$ & 932.05695 & $0.021^{*}$ & 2.86 & -0.54 \\
\hline & & $11 \mathrm{P}(34)$ & 939.61272 & 0.013 & 3.56 & -0.55 \\
\hline & $\mathrm{PH}_{3}$ & $10 \mathrm{P}(32)$ & 1059.15808 & 17.3 & 0.58 & -0.87 \\
\hline & $\mathrm{HNO}_{3}$ & $11 \mathrm{P}(34)$ & 939.61272 & 0.224 & 2.39 & 0.20 \\
\hline & & $11 \mathrm{P}(42)$ & 932.05695 & $0.756^{*}$ & 2.57 & 0.03 \\
\hline & $\mathrm{SF}_{6}$ & $11 \mathrm{P}(22)$ & 950.14015 & 476 & -0.33 & -0.21 \\
\hline & $\mathrm{CH}_{3} \mathrm{OH}$ & $10 \mathrm{P}(40)$ & 1052.26317 & 45.8 & 0.42 & -0.22 \\
\hline & $\mathrm{HCOOH}$ & $10 \mathrm{R}(34)$ & 1104.91182 & 228 & -0.56 & -0.26 \\
\hline & OCS & $10 \mathrm{P}(28)$ & 1062.49464 & 2.06 & 0.54 & -0.58 \\
\hline & $\mathrm{CH}_{3} \mathrm{CN}$ & $10 \mathrm{P}(32)$ & 1059.15808 & 3.74 & -0.26 & -0.30 \\
\hline & $\mathrm{SO}_{2}$ & 10R(36) & 1105.94358 & 1.73 & 0.80 & -0.12 \\
\hline & $\mathrm{H}_{2} \mathrm{O}$ & $11 \mathrm{P}(24)$ & 948.45233 & 0.000910 & 4.62 & 0.79 \\
\hline
\end{tabular}


TABLE 4: Continued.

\begin{tabular}{|c|c|c|c|c|c|c|}
\hline Laser & M & Transition & $v, \mathrm{~cm}^{-1}$ & $\sigma(\mathrm{M})^{\mathrm{a}}$ & $K_{T}$ & $K_{P}$ \\
\hline \multirow{14}{*}{46} & $\mathrm{NH}_{3}$ & 9P(18) & 967.44673 & 151 & -0.95 & -0.13 \\
\hline & $\mathrm{C}_{2} \mathrm{H}_{4}$ & $9 \mathrm{P}(36)$ & 949.82361 & 75.5 & 0.55 & -0.07 \\
\hline & $\mathrm{CO}_{2}$ & $9 \mathrm{P}(38)$ & 947.72257 & 0.009 & 6.83 & -0.85 \\
\hline & $\mathrm{O}_{3}$ & $9 \mathrm{R}(38)$ & 1007.32003 & 14.4 & 1.51 & -0.20 \\
\hline & $\mathrm{NO}_{2}$ & $10 \mathrm{R}(18)$ & 880.14964 & 0.617 & 1.99 & -0.45 \\
\hline & $\mathrm{PH}_{3}$ & $9 \mathrm{R}(12)$ & 992.16155 & 20.1 & -0.75 & 0.42 \\
\hline & $\mathrm{HNO}_{3}$ & $10 \mathrm{R}(16)$ & 878.74397 & 116 & 0.00 & -0.16 \\
\hline & $\mathrm{SF}_{6}$ & $9 \mathrm{P}(38)$ & 947.72257 & 860 & -0.01 & -0.57 \\
\hline & $\mathrm{CH}_{3} \mathrm{OH}$ & $9 \mathrm{R}(36)$ & 1006.33091 & 31.1 & 0.96 & -0.19 \\
\hline & $\mathrm{HCOOH}$ & $9 \mathrm{R}(40)$ & 1008.28028 & 0.572 & 0.85 & -0.04 \\
\hline & OCS & $10 \mathrm{R}(14)$ & 877.32170 & 2.41 & 1.16 & -0.59 \\
\hline & $\mathrm{CH}_{3} \mathrm{CN}$ & $9 \mathrm{R}(16)$ & 994.82189 & 0.839 & 1.88 & -0.47 \\
\hline & $\mathrm{C}_{2} \mathrm{H}_{6}$ & $10 \mathrm{P}(4)$ & 862.98995 & 0.666 & 0.98 & -0.18 \\
\hline & $\mathrm{H}_{2} \mathrm{O}$ & $11 \mathrm{R}(16)$ & 878.74397 & 0.000532 & 4.97 & 0.77 \\
\hline \multirow{20}{*}{38} & $\mathrm{NH}_{3}$ & $10 \mathrm{R}(10)$ & 1034.18567 & 86.9 & -0.39 & -0.22 \\
\hline & $\mathrm{C}_{2} \mathrm{H}_{4}$ & $11 \mathrm{R}(34)$ & 949.30088 & 121 & 0.14 & -0.19 \\
\hline & $\mathrm{CO}_{2}$ & $10 \mathrm{R}(32)$ & 1046.81398 & 0.011 & 6.41 & -0.50 \\
\hline & $\mathrm{O}_{3}$ & $10 \mathrm{R}(14)$ & 1036.67254 & 33.4 & -0.20 & -0.28 \\
\hline & & $10 \mathrm{R}(8)$ & 1032.91023 & $38.3^{*}$ & -0.22 & -0.39 \\
\hline & & $10 \mathrm{R}(40)$ & 1050.77747 & $50.5^{*}$ & -0.62 & -0.15 \\
\hline & $\mathrm{NO}_{2}$ & 11P(16) & 916.81456 & 0.134 & 2.86 & -0.36 \\
\hline & $\mathrm{PH}_{3}$ & $10 \mathrm{R}(22)$ & 1041.39099 & 11.3 & -0.29 & -0.65 \\
\hline & & $10 \mathrm{R}(6)$ & 1031.61345 & $13.9^{*}$ & -0.57 & -0.29 \\
\hline & $\mathrm{HNO}_{3}$ & $11 \mathrm{P}(38)$ & 898.25437 & 35.3 & 0.43 & -0.07 \\
\hline & & $11 \mathrm{P}(40)$ & 896.42949 & $53.4^{*}$ & -0.16 & -0.03 \\
\hline & $\mathrm{SF}_{6}$ & $11 \mathrm{R}(30)$ & 947.29249 & 641 & -0.06 & -0.16 \\
\hline & $\mathrm{CH}_{3} \mathrm{OH}$ & $10 \mathrm{R}(10)$ & 1034.18567 & 66.3 & 0.16 & -0.04 \\
\hline & & $10 \mathrm{R}(8)$ & 1032.91023 & $81.5^{*}$ & 0.79 & -0.02 \\
\hline & $\mathrm{HCOOH}$ & $10 \mathrm{R}(24)$ & 1042.51764 & 2.50 & 1.22 & -0.28 \\
\hline & & $10 \mathrm{R}(40)$ & 1050.77747 & $4.01^{*}$ & 1.15 & -0.25 \\
\hline & OCS & $10 \mathrm{R}(12)$ & 1035.43977 & 1.98 & 0.08 & -0.52 \\
\hline & $\mathrm{CH}_{3} \mathrm{CN}$ & $10 \mathrm{R}(34)$ & 1047.83586 & 3.57 & -0.10 & -0.62 \\
\hline & $\mathrm{SO}_{2}$ & $10 \mathrm{R}(46)$ & 1053.53509 & 0.024 & 4.78 & 0.00 \\
\hline & $\mathrm{H}_{2} \mathrm{O}$ & $11 \mathrm{R}(32)$ & 948.30848 & 0.00577 & 5.06 & -0.34 \\
\hline \multirow{16}{*}{48} & $\mathrm{NH}_{3}$ & $9 \mathrm{P}(20)$ & 967.55794 & 79.2 & -1.48 & 0.56 \\
\hline & $\mathrm{C}_{2} \mathrm{H}_{4}$ & $9 \mathrm{P}(40)$ & 950.27719 & 38.0 & 0.31 & 0.19 \\
\hline & $\mathrm{CO}_{2}$ & 9P(18) & 969.16198 & 0.008 & 6.48 & -0.83 \\
\hline & $\mathrm{O}_{3}$ & $9 \mathrm{R}(32)$ & 1002.18855 & 11.4 & 2.01 & -0.15 \\
\hline & & $9 \mathrm{R}(40)$ & 1006.00000 & $11.8^{*}$ & 1.68 & -0.22 \\
\hline & $\mathrm{NO}_{2}$ & $10 \mathrm{P}(12)$ & 880.59011 & 0.159 & 1.00 & 0.08 \\
\hline & $\mathrm{PH}_{3}$ & 9R(14) & 992.26858 & 21.3 & -0.08 & -0.29 \\
\hline & $\mathrm{HNO}_{3}$ & $10 \mathrm{P}(14)$ & 879.08844 & 90.0 & -0.53 & -0.08 \\
\hline & $\mathrm{SF}_{6}$ & $9 \mathrm{P}(40)$ & 950.27719 & 476 & -0.23 & -0.23 \\
\hline & & $9 \mathrm{P}(42)$ & 948.42788 & $484^{*}$ & -0.68 & 0.20 \\
\hline & $\mathrm{CH}_{3} \mathrm{OH}$ & $9 \mathrm{R}(36)$ & 1004.13958 & 28.9 & 1.23 & -0.25 \\
\hline & $\mathrm{HCOOH}$ & 9R(34) & 1003.17544 & 0.365 & 1.63 & -0.06 \\
\hline & OCS & $10 \mathrm{P}(30)$ & 866.40889 & 6.03 & -0.43 & -0.39 \\
\hline & $\mathrm{CH}_{3} \mathrm{CN}$ & $10 \mathrm{R}(28)$ & 907.29104 & 0.559 & -0.06 & -0.24 \\
\hline & $\mathrm{C}_{2} \mathrm{H}_{6}$ & $10 \mathrm{P}(36)$ & 861.34626 & 0.777 & 0.46 & -0.02 \\
\hline & $\mathrm{H}_{2} \mathrm{O}$ & $11 \mathrm{P}(24)$ & 871.30294 & 0.00549 & 4.02 & -0.29 \\
\hline
\end{tabular}


TABLE 4: Continued.

\begin{tabular}{|c|c|c|c|c|c|c|}
\hline Laser & M & Transition & $v, \mathrm{~cm}^{-1}$ & $\sigma(\mathrm{M})^{\mathrm{a}}$ & $K_{T}$ & $K_{P}$ \\
\hline \multirow{15}{*}{268} & $\mathrm{NH}_{3}$ & $10 \mathrm{P}(32)$ & 1046.35094 & 264 & -0.52 & -0.64 \\
\hline & $\mathrm{C}_{2} \mathrm{H}_{4}$ & $11 \mathrm{P}(21)$ & 949.43752 & 149 & 0.23 & -0.30 \\
\hline & $\mathrm{CO}_{2}$ & 10R(11) & 1081.07931 & 0.013 & 7.17 & -0.95 \\
\hline & $\mathrm{O}_{3}$ & $10 \mathrm{P}(27)$ & 1050.83825 & 56.6 & -0.54 & -0.26 \\
\hline & $\mathrm{NO}_{2}$ & $11 \mathrm{P}(37)$ & 934.69411 & 0.067 & 3.71 & 0.03 \\
\hline & $\mathrm{PH}_{3}$ & $11 R(36)$ & 988.95058 & 19.8 & 1.33 & -0.21 \\
\hline & & $11 R(42)$ & 991.76694 & $38.6^{*}$ & -1.11 & 0.02 \\
\hline & $\mathrm{HNO}_{3}$ & $11 \mathrm{P}(50)$ & 921.46702 & 6.33 & 2.00 & -0.04 \\
\hline & $\mathrm{SF}_{6}$ & $11 \mathrm{P}(23)$ & 947.68595 & 851 & 0.00 & -0.55 \\
\hline & $\mathrm{CH}_{3} \mathrm{OH}$ & $10 \mathrm{P}(46)$ & 1033.08142 & 95.5 & 0.73 & -0.08 \\
\hline & $\mathrm{HCOOH}$ & $10 \mathrm{R}(24)$ & 1089.24975 & 77.9 & -0.33 & -0.13 \\
\hline & OCS & $10 \mathrm{P}(16)$ & 1060.23019 & 2.20 & 0.14 & -0.60 \\
\hline & $\mathrm{CH}_{3} \mathrm{CN}$ & $10 \mathrm{P}(24)$ & 1053.46560 & 2.92 & -0.12 & -0.55 \\
\hline & $\mathrm{SO}_{2}$ & $10 \mathrm{R}(36)$ & 1095.95040 & 1.03 & 1.38 & -0.22 \\
\hline & $\mathrm{H}_{2} \mathrm{O}$ & 9R(16) & 1084.33484 & 0.00439 & 5.37 & 0.21 \\
\hline
\end{tabular}

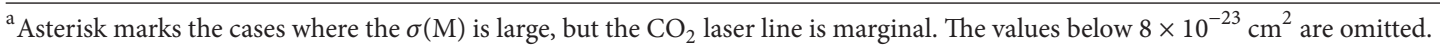

TABLE 5: Comparison of our calculation results with the experimental literature data for the strongest transitions in $\mathrm{NH}_{3}, \mathrm{C}_{2} \mathrm{H}_{4}$, and $\mathrm{O}_{3}$ molecules at ${ }^{12} \mathrm{C}^{16} \mathrm{O}_{2}$ laser frequencies.

\begin{tabular}{|c|c|c|c|c|c|c|c|c|c|}
\hline \multirow{2}{*}{ M } & \multirow{2}{*}{$\begin{array}{l}\text { Laser } \\
\text { line }\end{array}$} & \multirow{2}{*}{$v, \mathrm{~cm}^{-1}$} & \multicolumn{3}{|c|}{$\sigma(\mathrm{M}), 10^{-20} \mathrm{~cm}^{2}$} & \multicolumn{2}{|c|}{$K_{T}$} & \multicolumn{2}{|c|}{$K_{P}$} \\
\hline & & & {$[24]$} & {$[22]$} & $\mathrm{a}$ & {$[22]$} & $\mathrm{a}$ & {$[22]$} & $\mathrm{a}$ \\
\hline \multirow{7}{*}{$\mathrm{NH}_{3}$} & 9R(16) & 1075.98782 & 51.6 & $\mathrm{c}$ & 52.1 & $\mathrm{c}$ & 0.70 & $c$ & -0.29 \\
\hline & $11 \mathrm{R}(8)$ & 967.70723 & 88.9 & 92.6 & 102 & -1.07 & -0.98 & -0.30 & -0.33 \\
\hline & $11 \mathrm{R}(14)$ & 971.93026 & $2.56^{\mathrm{b}}$ & 30.2 & 27.6 & -0.62 & -0.96 & -0.59 & -0.61 \\
\hline & $11 \mathrm{P}(32)$ & 932.96042 & 60.9 & $c$ & 57.6 & c & 0.03 & c & -0.44 \\
\hline & $11 \mathrm{P}(34)$ & 931.00143 & 50.3 & c & 55.2 & c & -0.87 & c & 0.60 \\
\hline & $9 \mathrm{R}(30)$ & 1084.63514 & $c$ & c & 303 & c & 0.38 & c & -0.83 \\
\hline & $9 \mathrm{P}(20)$ & 1046.85423 & 8.77 & 12.1 & 13.2 & -1.50 & -1.62 & 0.96 & 0.92 \\
\hline \multirow{4}{*}{$\mathrm{C}_{2} \mathrm{H}_{4}$} & $11 \mathrm{P}(14)$ & 949.47931 & 118.4 & 145.1 & 133 & -0.54 & 0.16 & 0.03 & -0.13 \\
\hline & $11 \mathrm{P}(16)$ & 947.74198 & $c$ & 23.9 & 21.4 & -0.46 & -0.55 & -0.02 & 0.01 \\
\hline & $11 \mathrm{R}(24)$ & 978.47229 & 20.5 & 20.9 & 19.3 & -0.83 & -0.49 & -0.06 & -0.02 \\
\hline & $11 \mathrm{P}(12)$ & 951.19226 & $\mathrm{c}$ & 19.7 & 17.3 & -0.01 & -0.46 & 0.30 & 0.42 \\
\hline \multirow{3}{*}{$\mathrm{O}_{3}$} & $9 \mathrm{P}(14)$ & 1052.19555 & 51.6 & $\mathrm{c}$ & 47.4 & $c$ & -0.62 & $c$ & 0.08 \\
\hline & $9 \mathrm{P}(12)$ & 1053.92350 & 49.5 & c & 44.0 & c & -0.38 & c & -0.22 \\
\hline & $9 \mathrm{P}(8)$ & 1057.30016 & 51.6 & c & 49.2 & c & -0.02 & c & -0.12 \\
\hline
\end{tabular}

${ }^{\mathrm{a}}$ This work.

${ }^{\mathrm{b}}$ Probably, misprint.

${ }^{\mathrm{c}}$ No data.

\section{Discussion}

Some of our results are compared with the experimental literature data in Table 5. As one can see from the table, the present results agree favorably with the experimental data of Patty et al. [24] and Persson et al. [22], who have determined $\sigma, K_{P}$, and $K_{T}$ coefficients for 26-laser absorption by $\mathrm{NH}_{3}$, $\mathrm{O}_{3}$, and $\mathrm{C}_{2} \mathrm{H}_{4}$ molecules.

Note that only in several cases our $\mathrm{M}$ molecules are important as "standard" air pollutant $\left(\mathrm{NH}_{3}, \mathrm{C}_{2} \mathrm{H}_{4}, \mathrm{PH}_{3}\right.$, and
$\mathrm{O}_{3}$ ) and in other cases our $\mathrm{M}$ molecules may happen in the air only near special industrial objects. As one can see from Tables 1-4, 26-laser is a good choice for all these four gases.

There are several advantages of the other $\mathrm{CO}_{2}$ lasers: monitoring of $\mathrm{HNO}_{3}, \mathrm{NO}_{2}, \mathrm{C}_{2} \mathrm{H}_{6}$, and $\mathrm{CO}_{2}$ molecules requires 46-/48-/36-, 36-, 36-/46-/48-, and 38-/268-lasers, respectively, instead of 26-laser.

We included in Table 4 several molecules with low cross sections ( $\mathrm{OCS}, \mathrm{CH}_{3} \mathrm{CN}, \mathrm{C}_{2} \mathrm{H}_{6}, \mathrm{SO}_{2}$, and $\mathrm{NO}_{2}$ ). Although $\mathrm{CO}_{2}$ laser is not the best choice to detect these molecules, 
TABLE 6: The largest absorption cross sections (in $10^{-20} \mathrm{~cm}^{2}$ units) of $\mathrm{CH}_{3} \mathrm{OH}$ and $\mathrm{HCCOH}$ at low pressure and $T=296 \mathrm{~K}$. The Doppler shape of spectral lines is assumed.

\begin{tabular}{|c|c|c|c|c|}
\hline M & Laser & Line & $v, \mathrm{~cm}^{-1}$ & $\sigma(\mathrm{M})^{\mathrm{a}}$ \\
\hline \multirow{11}{*}{$\mathrm{CH}_{3} \mathrm{OH}$} & 26 & $10 \mathrm{P}(34)$ & 1033.48800 & 126 \\
\hline & 36 & $10 \mathrm{P}(16)$ & 1004.27987 & 67.2 \\
\hline & 28 & $10 \mathrm{P}(22)$ & 1067.35888 & 93.0 \\
\hline & 38 & $10 \mathrm{R}(8)$ & 1032.91023 & $200^{*}$ \\
\hline & 38 & $10 \mathrm{R}(40)$ & 1050.77747 & $193^{*}$ \\
\hline & 38 & $10 \mathrm{P}(36)$ & 998.62350 & 113 \\
\hline & 46 & $9 \mathrm{R}(46)$ & 1010.98950 & $44.7^{*}$ \\
\hline & 46 & 9R(28) & 1002.08279 & 19.4 \\
\hline & 48 & 9R(40) & 1006.00000 & $146^{*}$ \\
\hline & 48 & 9R(24) & 998.01156 & 107 \\
\hline & 268 & $10 \mathrm{P}(27)$ & 1050.83825 & 368 \\
\hline \multirow{10}{*}{$\mathrm{HCOOH}$} & 26 & 10R(18) & 1077.30252 & 186 \\
\hline & 26 & $10 \mathrm{R}(28)$ & 1083.47878 & 189 \\
\hline & 26 & $10 \mathrm{R}(40)$ & 1090.02837 & $976^{*}$ \\
\hline & 36 & $10 \mathrm{R}(28)$ & 1037.16713 & 9.59 \\
\hline & 28 & $10 \mathrm{P}(8)$ & 1078.04242 & 327 \\
\hline & 28 & $10 \mathrm{R}(34)$ & 1104.91182 & 285 \\
\hline & 38 & $10 \mathrm{R}(44)$ & 1052.63615 & 19.3 \\
\hline & 46 & 9R(26) & 1000.94716 & 2.64 \\
\hline & 48 & 9R(30) & 1001.17880 & 2.74 \\
\hline & 268 & 10R(19) & 1086.22023 & 118 \\
\hline
\end{tabular}

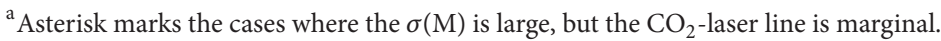

these data may be useful in special cases, for example, when $\mathrm{CO}_{2}$ LIDAR is used to monitor the leakage of these gases from industrial areas.

Surely, the data in all our tables are only starting points in discussion about applicability of particular $\mathrm{CO}_{2}$-laser transitions for remote sensing under atmospheric conditions, because at many wavenumbers, the absorption by $\mathrm{H}_{2} \mathrm{O}$ may be much stronger than absorption by the gases of interest. Hence one always should find the tradeoff between the absorption of $\mathrm{H}_{2} \mathrm{O}$ and the absorption of these gases.

For example, the "best" line for $\mathrm{NH}_{3}$ detection by 26laser in Table 4 is $10 \mathrm{R}(30)$ with $\sigma\left(\mathrm{NH}_{3}\right)=303 \times 10^{-20} \mathrm{~cm}^{2}$. However, at this wavelength, the ratio $\sigma\left(\mathrm{NH}_{3}\right) / \sigma\left(\mathrm{H}_{2} \mathrm{O}\right)$ is only $4.4 \times 10^{5}$. If we choose another 26-laser line for $\mathrm{NH}_{3}$ detection, $\mathrm{P}(34)\left(931.0014 \mathrm{~cm}^{-1}\right)$ with $\sigma\left(\mathrm{NH}_{3}\right)=55.2 \times$ $10^{-20} \mathrm{~cm}^{2}$ and $\sigma\left(\mathrm{H}_{2} \mathrm{O}\right)=0.0023 \times 10^{-23} \mathrm{~cm}^{2}$, the ratio will be much higher: $\sigma\left(\mathrm{NH}_{3}\right) / \sigma\left(\mathrm{H}_{2} \mathrm{O}\right)=2.4 \times 10^{7}$. Hence, this another 26-laser line is better for $\mathrm{NH}_{3}$ detection, although the value $\sigma\left(\mathrm{NH}_{3}\right)$ is lower.

Therefore, we included $\mathrm{H}_{2} \mathrm{O}$ in our calculations; see the results in Tables 1,2 , and 3. Our $\sigma\left(\mathrm{H}_{2} \mathrm{O}\right)$ data in the tables should help to choose the "best" pairs of $\mathrm{CO}_{2}$-laser lines (absorbing and nonabsorbing) for remote sensing of the gases of interest. Note that the pair of $\mathrm{CO}_{2}$-laser wavenumbers may originate from two isotopically different $\mathrm{CO}_{2}$ lasers; therefore the possibility to use many isotopic variations of $\mathrm{CO}_{2}$ laser simplifies strongly the choice of such pairs.

\section{Application to FIR Lasers}

There are several important benchmark molecules which are normally used in $\mathrm{CO}_{2}$-laser-pumped FIR lasers: $\mathrm{CH}_{3} \mathrm{OH}$, $\mathrm{CH}_{2} \mathrm{~F}_{2}, \mathrm{HCOOH},{ }^{15} \mathrm{NH}_{3}, \mathrm{CD}_{3} \mathrm{OD}, \mathrm{CD}_{3} \mathrm{OH}, \mathrm{CD}_{3} \mathrm{Cl},{ }^{13} \mathrm{CD}_{3} \mathrm{I}$, and ${ }^{13} \mathrm{CH}_{3} \mathrm{~F}$. The absorption of $\mathrm{CO}_{2}$ radiation by these molecules results in FIR-laser emission. Table 6 lists our $\sigma(\mathrm{M})$ values for $\mathrm{CH}_{3} \mathrm{OH}$ and $\mathrm{HCOOH}$ at low pressures, where the shapes of spectral lines of these molecules are given by Doppler effect. As one can see, there are a lot of interesting possibilities to obtain new strong sources of FIR radiation. One of them may be 9R(19) line of 268-laser, which has very large $\sigma(\mathrm{HCOOH})$ value.

Although there is no direct relation between intensities of $\mathrm{CO}_{2}$ absorption and FIR emission, it is clear that using 1000 $\mathrm{CO}_{2}$-laser lines instead of 100-200 should increase strongly the amount of strong FIR-laser transitions.

\section{Conclusion}

The absorption cross sections and $K_{T}$ and $K_{P}$ parameters of some molecules $\left(\mathrm{NH}_{3}, \mathrm{C}_{2} \mathrm{H}_{4}, \mathrm{CO}_{2}, \mathrm{O}_{3}, \mathrm{NO}_{2}, \mathrm{PH}_{3}, \mathrm{HNO}_{3}\right.$, $\mathrm{SF}_{6}, \mathrm{CH}_{3} \mathrm{OH}, \mathrm{HCOOH}, \mathrm{OCS}, \mathrm{CH}_{3} \mathrm{CN}, \mathrm{C}_{2} \mathrm{H}_{6}, \mathrm{SO}_{2}$, and $\left.\mathrm{H}_{2} \mathrm{O}\right)$ at $\mathrm{CO}_{2}$ laser frequencies $\left({ }^{12} \mathrm{C}^{16} \mathrm{O}_{2},{ }^{13} \mathrm{C}^{16} \mathrm{O}_{2},{ }^{12} \mathrm{C}^{18} \mathrm{O}_{2}\right.$, ${ }^{14} \mathrm{C}^{16} \mathrm{O}_{2},{ }^{14} \mathrm{C}^{18} \mathrm{O}_{2},{ }^{13} \mathrm{C}^{18} \mathrm{O}_{2}$, and ${ }^{12} \mathrm{C}^{16} \mathrm{O}^{18} \mathrm{O}$ ) have been calculated with the use of spectroscopical parameters from GEISA database. The present results are in reasonable agreement 
with other experimental measurements for $\mathrm{NH}_{3}, \mathrm{O}_{3}$, and $\mathrm{C}_{2} \mathrm{H}_{4}$. The results of the calculations may be used in designing the differential absorption technique for remote monitoring of these molecules.

\section{References}

[1] C. Weitkamp, Lidar Range-Resolved Optical Remote Sencing of the Atmosphere, Springer Science and Business Media Inc., 2005.

[2] B. I. Vasil'ev and O. M. Mannoun, "IR differential-absorption lidars for ecological monitoring of the environment," Quantum Electronics, vol. 36, no. 9, pp. 801-820, 2006.

[3] J. R. Quagliano, P. O. Stoutland, R. R. Petrin et al., "Quantitative chemical identification of four gases in remote infrared (9-11 $\mu \mathrm{m})$ differential absorption lidar experiments," Applied Optics, vol. 36, no. 9, pp. 1915-1927, 1997.

[4] S. Lundqvist, C. O. Falt, U. Persson, B. Marthinsson, and S. T. Eng, "Air pollution monitoring with a Q-switched $\mathrm{CO}_{2}$-laser lidar using heterodyne detection," Applied Optics, vol. 20, no. 14, pp. 2534-2538, 1981.

[5] U. Persson, S. Lundqvist, B. Marthinsson, and S. T. Eng, "Computerautomated $\mathrm{CO}_{2}$-laser long-path absorption system for air quality monitoring in the working environment," Applied Optics, vol. 23, no. 7, pp. 998-1002, 1984.

[6] W. Schnell and G. Fischer, "Carbon dioxide laser absorption coefficients of various air pollutants," Applied Optics, vol. 14, no. 9, pp. 2058-2059, 1975.

[7] A. Mayer, J. Comera, H. Charpentier, and C. Jaussaud, "Absorption coefficients of various pollutant gases at $\mathrm{CO}_{2}$ laser wavelengths, application to the remote sensing of those pollutants: errata," Applied Optics, vol. 17, no. 3, pp. 391-393, 1978.

[8] A. Pal, C. D. Clark, M. Sigman, and D. K. Killinger, "Differential absorption lidar $\mathrm{CO}_{2}$ laser system for remote sensing of TATP related gases," Applied Optics, vol. 48, no. 4, pp. B145-B150, 2009.

[9] K. I. Arshinov, M. K. Arshinov, V. V. Nevdakh, M.-Y. Perrin, A. Soufiani, and V. V. Yasnov, "Accuracy in determination of the temperature and partial pressure of $\mathrm{CO}_{2}$ in $\mathrm{CO}_{2}: \mathrm{N}_{2}: \mathrm{H}_{2} \mathrm{O}: \mathrm{NO}_{2}$ mixtures by multiple-frequency laser probing," Journal of Applied Spectroscopy, vol. 74, no. 6, pp. 903-909, 2007.

[10] M. Hamza, M. H. S. El-Ahl, and A. M. Hamza, "New laser system for sensitive remote sensing of ammonia in human breath," in Proceedings of the Air Monitoring and Detection of Chemical and Biological Agents II, vol. 3855 of Proceedings of SPIE, pp. 28-33, September 1999.

[11] L. Fiorani, F. Colao, and A. Palucci, "Measurement of Mount Etna plume by $\mathrm{CO}_{2}$-laser-based lidar," Optics Letters, vol. 34, no. 6, pp. 800-802, 2009.

[12] L. Fiorani, F. Colao, A. Palucci, D. Poreh, A. Aiuppa, and G. Giudice, "First-time lidar measurement of water vapor flux in a volcanic plume," Optics Communications, vol. 284, no. 5, pp. 1295-1298, 2011.

[13] P. P. Geiko and A. Tikhomirov, "Remote measurement of chemical warfare agents by differential absorption $\mathrm{CO}_{2}$ lidar," Optical Memory and Neural Networks, vol. 20, no. 1, pp. 71-75, 2011.

[14] E. M. Telles, H. Odashima, L. R. Zink, and K. M. Evenson, "Optically pumped FIR laser lines from $\mathrm{CH}_{3} \mathrm{OH}$ : new laser lines, frequency measurements, and assignments," Journal of Molecular Spectroscopy, vol. 195, no. 2, pp. 360-366, 1999.
[15] C. Bellecci, M. Francucci, P. Gaudio et al., "Application of a $\mathrm{CO}_{2}$ dial system for infrared detection of forest fire and reduction of false alarm," Applied Physics B, vol. 87, no. 2, pp. 373-378, 2007.

[16] P. Gaudio, M. Gelfusa, I. Lupelli et al., "First open field measurements with a portable $\mathrm{CO}_{2}$ lidar/ dial system for early forest fires detection," in Lidar Technologies, Techniques, and Measurements for Atmospheric Remote Sensing VII, September 2011.

[17] N. Jacquinet-Husson, L. Crepeau, R. Armante et al., "The 2009 edition of the GEISA spectroscopic database," Journal of Quantitative Spectroscopy and Radiative Transfer, vol. 112, no. 15, pp. 2395-2445, 2011.

[18] L. F. Chernogor and A. S. Rashkevich, "Results of en-route monitoring of the laser gas polluting impurities in the atmosphere," Eastern European Journal of Enterprise Technologies, vol. 52, article 57, 1987.

[19] K. Fox, "Strengths of the SF6 transitions pumped by a $\mathrm{CO}_{2}$ laser," Optics Communications, vol. 19, no. 3, pp. 397-400, 1976.

[20] J. L. Lyman, R. G. Anderson, R. A. Fisher, and B. J. Feldman, "Absorption of pulsed $\mathrm{CO}_{2}$-laser radiation by SF6 at $140 \mathrm{~K}$," Optics Letters, vol. 3, no. 6, pp. 238-240, 1978.

[21] H. Kariminezhad, P. Parvin, F. Borna, and A. Bavali, "SF6 leak detection of high-voltage installations using TEA- $\mathrm{CO}_{2}$ laserbased DIAL," Optics and Lasers in Engineering, vol. 48, no. 4, pp. 491-499, 2010.

[22] U. Persson, B. Marthinsson, J. Johansson, and S. T. Eng, "Temperature and pressure dependence of $\mathrm{NH}_{3}$ and $\mathrm{C}_{2} \mathrm{H}_{4}$ absorption cross sections at $\mathrm{CO}_{2}$ laser wavelengths," Applied Optics, vol. 19, no. 10, pp. 1711-1715, 1980.

[23] J. N. Olsen, "Laser-initiated channels for ion transport: $\mathrm{CO}_{2}$ laser absorption and heating of $\mathrm{NH}_{3}$ and $\mathrm{C}_{2} \mathrm{H}_{4}$ gases," Journal of Applied Physics, vol. 52, no. 5, pp. 3279-3285, 1981.

[24] R. R. Patty, G. M. Russwurm, W. A. McClenny, and D. R. Morgan, " $\mathrm{CO}_{2}$ laser absorption coefficients for determining ambient levels of $\mathrm{O}_{3}, \mathrm{NH}_{3}$, and $\mathrm{C}_{2} \mathrm{H}_{4}$," Applied Optics, vol. 13, no. 12, pp. 2850-2854, 1974.

[25] A. P. Force, D. K. Killinger, W. E. DeFeo, and N. Menyuk, "Laser remote sensing of atmospheric ammonia using a $\mathrm{CO}_{2}$ lidar system," Applied Optics, vol. 24, no. 17, pp. 2837-2841, 1985.

[26] Y.Zhao, "Line-pair selections for remote sensing of atmospheric ammonia by use of a coherent $\mathrm{CO}_{2}$ differential absorption lidar system," Applied Optics, vol. 39, no. 6, pp. 997-1007, 2000.

[27] B. D. Green and J. I. Steinfeld, "Absorption coefficients for fourteen gases at $\mathrm{CO}_{2}$-laser frequencies," Applied Optics, vol. 15, p. $1688,1975$.

[28] L. T. Molina and W. B. Grant, "FTIR-spectrometer-determined absorption coefficients of seven hydrazine fuel gasesimplications for laser remote sensing," Applied Optics, vol. 23, no. 21, pp. 3893-3900, 1983.

[29] N. Menyuk, D. K. Killinger, and W. E. DeFeo, "Laser remote sensing of hydrazine, MMH, and UDMH using a differentialabsorption $\mathrm{CO}_{2}$ lidar," Applied Optics, vol. 21, no. 12, pp. 22752286, 1982.

[30] J. S. Ryan, M. H. Hubert, and R. A. Crane, "Water vapor absorption at isotopic $\mathrm{CO}_{2}$ laser wavelengths," Applied Optics, vol. 22, no. 5, pp. 711-717, 1983.

[31] H. Ahlberg, S. Lundqvist, and S. T. Eng, "Absorption coefficients of chlorine-dioxide ${ }^{12} \mathrm{C}^{18} \mathrm{O}_{2}$ laser wavelengths: applications to remote monitoring in the working environment," Applied Optics, vol. 23, no. 17, pp. 2902-2905, 1984. 
[32] F. Allario and R. K. Seals Jr., "Measurements of $\mathrm{NH}_{3}$ absorption coefficients with a ${ }^{13} \mathrm{C}^{16} \mathrm{O}_{2}$ laser," Applied Optics, vol. 14, no. 9, pp. 2229-2233, 1975.

[33] Z. Zelinger, I. Jancik, and P. Engst, "Measurement of the $\mathrm{NH}_{3}$, $\mathrm{CCl}_{2} \mathrm{~F}_{2}, \mathrm{CHClF}_{2}, \mathrm{CFCl}_{3}$, and $\mathrm{CClF}_{3}$ absorption coefficients at isotopic ${ }^{13} \mathrm{C}^{16} \mathrm{O}_{2}$ laser wavelengths by photoacoustic spectroscopy," Applied Optics, vol. 31, p. 6974, 1992.

[34] D. Pereira, A. Scalabrin, G. P. Galvão, and K. M. Evenson, ${ }^{c 13} \mathrm{CD}^{3} \mathrm{OH}$ and ${ }^{12} \mathrm{CD}_{3} \mathrm{OH}$ optically pumped by a ${ }^{13} \mathrm{CO}_{2}$ laser: observations and assignments of FIR laser lines," International Journal of Infrared and Millimeter Waves, vol. 13, no. 4, pp. 497506, 1992.

[35] L. F. L. Costa, J. C. S. Moraes, F. C. Cruz, R. C. Viscovini, and D. Pereira, "Infrared and far-infrared spectroscopy of ${ }^{13} \mathrm{CH}_{3} \mathrm{OH}$ : teraHertz laser lines and assignments," Journal of Molecular Spectroscopy, vol. 241, no. 2, pp. 151-154, 2007.

[36] L. F. L. Costa, J. C. S. Moraes, F. C. Cruz, R. C. Viscovini, and D. Pereira, " $\mathrm{CH}_{3} \mathrm{OH}$ optically pumped by a ${ }^{13} \mathrm{CO}_{2}$ laser: new laser lines and assignments," Applied Physics B, vol. 86, no. 4, pp. 703-706, 2007.

[37] R. C. Viscovini, J. C. S. Moraes, L. F. L. Costa, F. C. Cruz, and D. Pereira, "DCOOD optically pumped by a ${ }^{13} \mathrm{CO}_{2}$ laser: new terahertz laser lines," Applied Physics B, vol. 91, no. 3-4, pp. 517520, 2008.

[38] J. C. Petersen and G. Duxbury, “Observation and assignment of submillimetre laser lines from $\mathrm{CH}_{3} \mathrm{OH}$ pumped by isotopic $\mathrm{CO}_{2}$ lasers," Applied Physics B, vol. 27, no. 1, pp. 19-25, 1982.

[39] J. C. Petersen and G. Duxbury, "Submillimetre laser lines from $\mathrm{CH}_{3} \mathrm{OH}$ pumped by ${ }^{13} \mathrm{C}^{18} \mathrm{O}_{2}$ pump laser: observations and assignments," Applied Physics B, vol. 34, no. 1, pp. 17-21, 1984.

[40] C. Freed, L. C. Bradley, and R. G. O’Donnell, "Absolute frequencies of lasing transitions in seven $\mathrm{CO}_{2}$ isotopic species," IEEE Journal of Quantum Electronics, vol. 16, no. 11, pp. 1195-1206, 1980. 

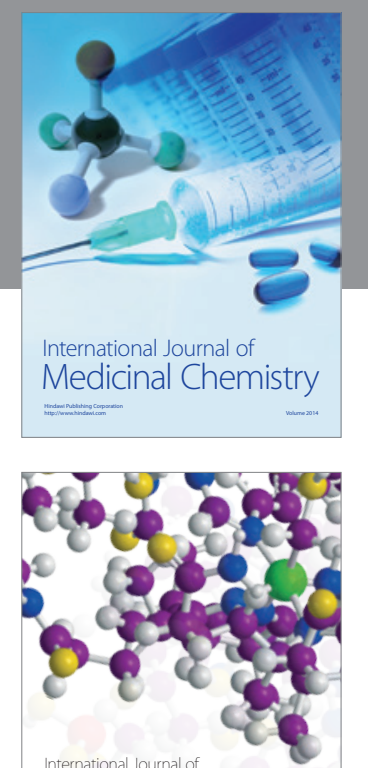

\section{Carbohydrate} Chemistry

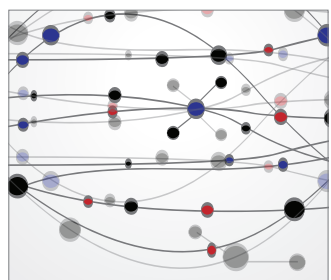

The Scientific World Journal
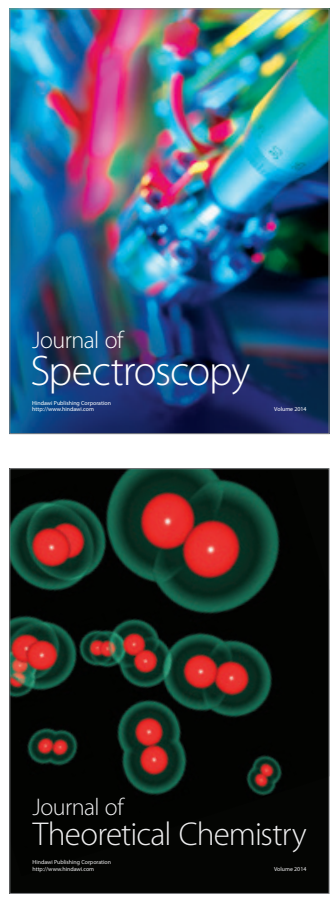
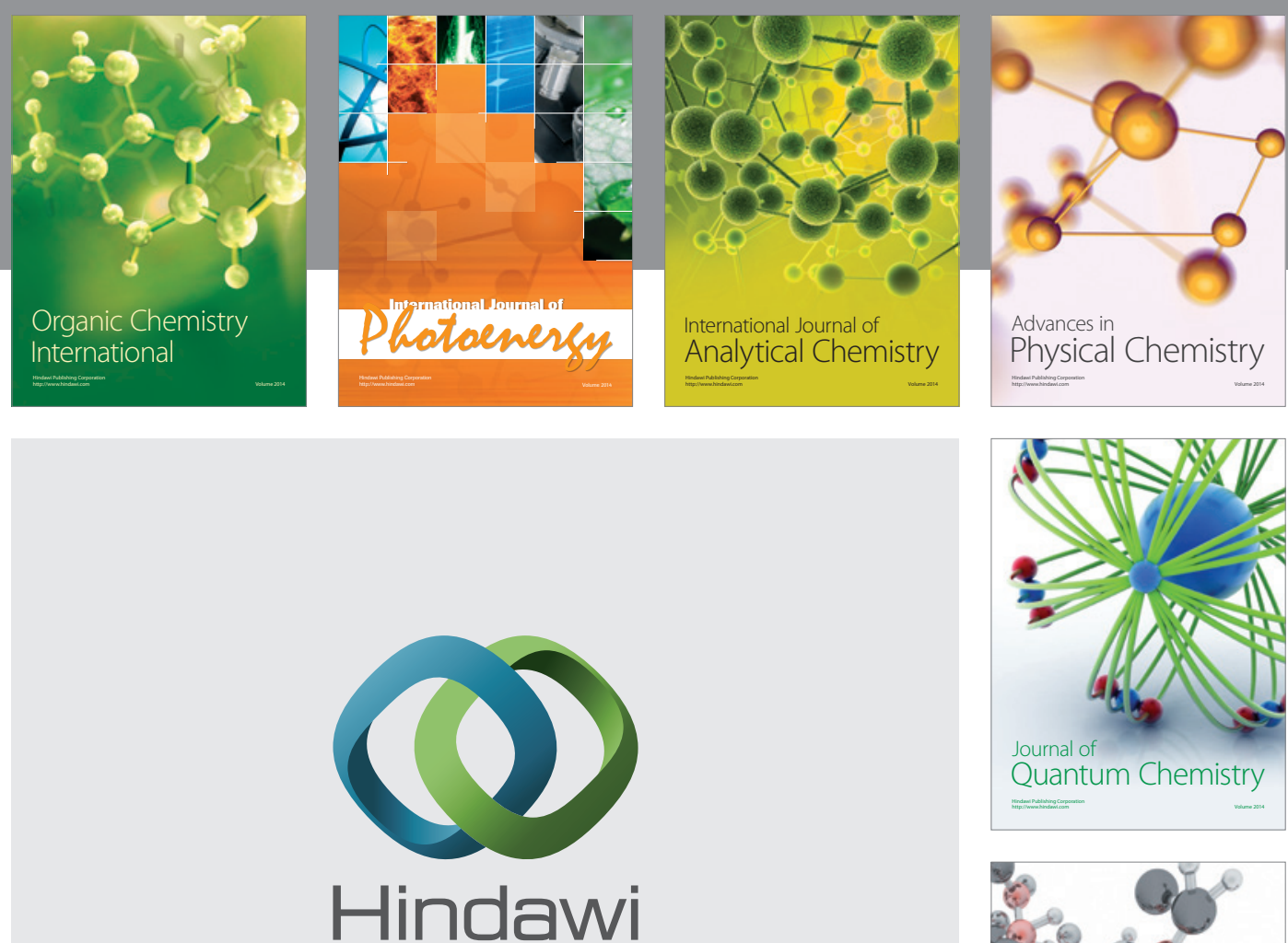

Submit your manuscripts at

http://www.hindawi.com

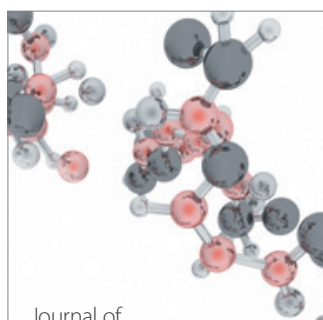

Analytical Methods

in Chemistry

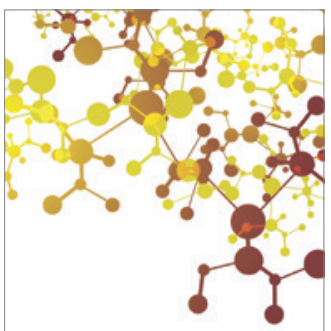

Journal of

Applied Chemistry

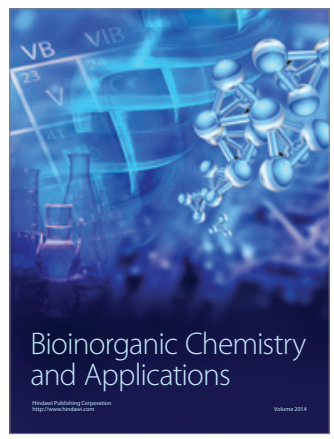

Inorganic Chemistry
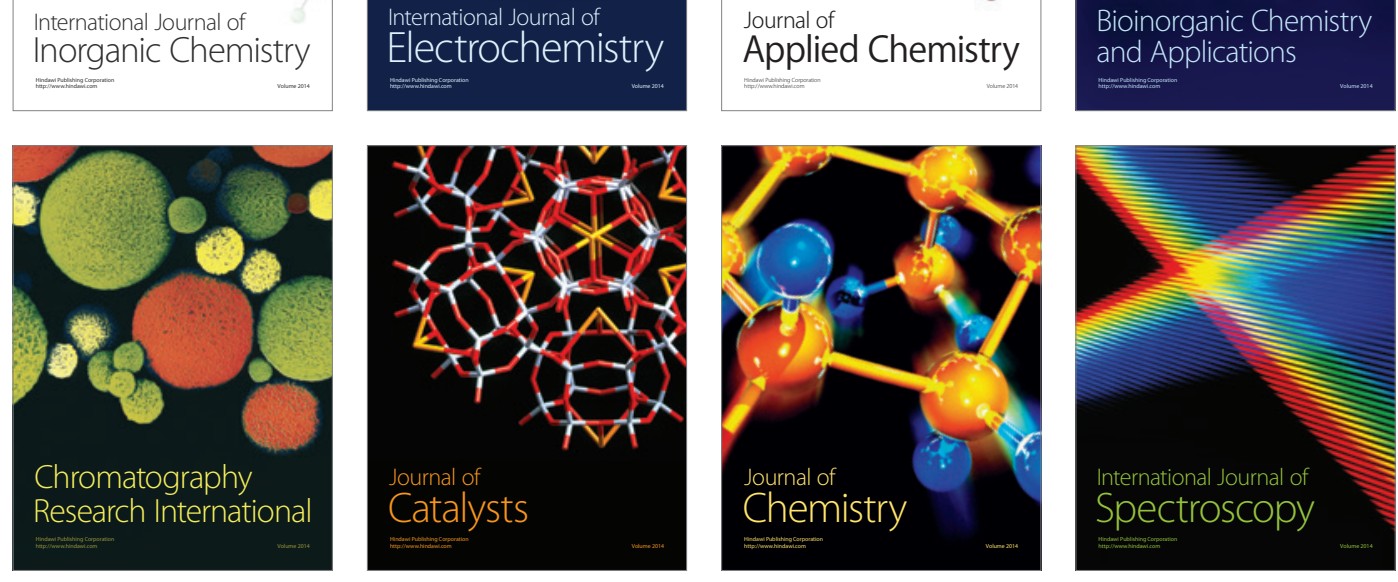